\title{
Selección de un modelo para evaluar la sostenibilidad hidroeléctrica mediante el método AHP
}

\author{
GÓMEZ ROMERO, JOSÉ ANDRÉS \\ Comisión Federal de Electricidad (México) \\ Correo electrónico: andresszz@hotmail.com \\ SOTO FLORES, ROCÍO \\ Escuela Superior de Comercio y Administración Unidad Santo Tomás \\ Instituto Politécnico Nacional (México) \\ Correo electrónico: mrsoto@ipn.mx \\ GARDUÑO ROMÁN, SUSANA \\ Instituto Politécnico Nacional (México) \\ Correo electrónico: sgarduno@ipn.mx
}

\begin{abstract}
RESUMEN
El desarrollo sostenible es un tema de interés desde hace más de tres décadas, derivado de lo cual, se han elaborado diversas soluciones que buscan implementar y controlar el desarrollo sostenible en las empresas. El sector de energía hidroeléctrica busca atender sus problemáticas ambientales y sociales a través de diversos modelos. El objetivo de este trabajo es seleccionar un modelo de desarrollo sostenible para evaluar la sostenibilidad hidroeléctrica mediante un método de toma de decisiones. Mediante el método proceso analítico jerárquico (AHP) y un grupo de enfoque se determinan las ponderaciones de los criterios, subcriterios y alternativas de los modelos de desarrollo sostenible. Una vez utilizado el método AHP se jerarquizaron los modelos de desarrollo sostenible, se determinó que el modelo del protocolo del IHA obtuvo la mayor ponderación, seguido de los modelos del BS 8900 y el Dow Jones Sustainability Indexes. En el análisis de sensibilidad se validó la selección del modelo del protocolo de la IHA. Los resultados presentados confirman que el protocolo de la IHA es el modelo de desarrollo sostenible más apropiado para evaluar la sostenibilidad hidroeléctrica, lo que proporciona apoyo a quienes buscan justificar su decisión de seleccionar un modelo de desarrollo sostenible, mediante métodos multicriterio.

Palabras clave: proceso analítico jerárquico, sostenibilidad hidroeléctrica, modelos de sostenibilidad, toma de decisiones multicriterio.
\end{abstract}

Clasificación JEL: C44; D79; Q01; Q25.

MSC2010: 90B50; 90C31.

Artículo recibido el 24 de enero de 2019 y aceptado el 15 de julio de 2019. 


\title{
Selection of a model to evaluate hydroelectric sustainability through the AHP method
}

\begin{abstract}
Sustainable development is the theme that has remained in the interest for more than three decades, as the result of which various solutions have been developed that seek to implement and control sustainability development in companies. The hydropower sector seeks to address its environmental and social problems through various models. The objective of this work is to select a sustainable development model to evaluate hydroelectric sustainability through a method of multicriteria decision making.

Through the method of Analytical Hierarchical Process (AHP), and a focus group it is possible to determine the weightings of the criteria, sub-criteria and alternatives of sustainable development models.

Once the AHP method was applied, the sustainable development models were hierarchized, it was found that the hydropower sustainability assessment protocol obtained the highest priority among the models, followed by the BS 8900 standard and the Dow Jones Sustainability Indexes (DJSI). In the sensitivity analysis, the selection of the hydropower sustainability assessment protocol model was validated, as it remained in the first place in three of the four proposed scenarios.

The results showed that the hydropower sustainability assessment protocol is the most appropriate sustainable development model to evaluate hydroelectric sustainability. This provides support to those who seek to justify their decision to select a sustainable development model, using multicriteria methods.
\end{abstract}

Keywords: analytical hierarchical process, sustainable hydropower, sustainability models, multi-criteria decision-making.

JEL classification: C44; D79; Q01; Q25.

MSC2010: 90B50; 90C31.

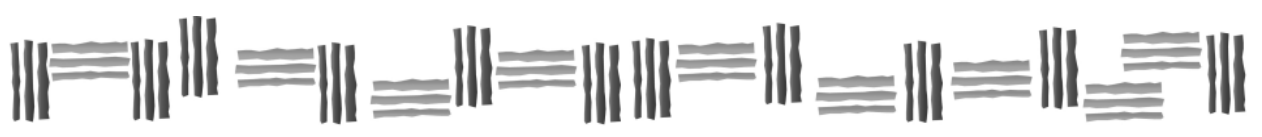




\section{Introducción.}

En la historia de la humanidad, numerosas culturas y civilizaciones reconocieron la necesidad de establecer relaciones holísticas entre la sociedad, la economía y el medio ambiente. Esto se incrementó con la Revolución Industrial y los cambios que de ahí se derivaron, como el acelerado avance tecnológico y científico y la espiral de adelantos que han llevado a la humanidad a cambios intensos que impactaron positiva y negativamente. De estos cambios han surgido nuevas tendencias y políticas, entre las que destaca el desarrollo sostenible que emana de un antiguo principio de la cultura humana, como es el respeto al entorno y a la relación ser humano-naturaleza (Quintana, 2011).

En 1987, la Comisión Mundial sobre Medio Ambiente y Desarrollo elaboró el informe Brundtland, titulado Nuestro Futuro Común, donde se formalizó el concepto de desarrollo sostenible como aquel que satisface las necesidades del presente, sin comprometer la capacidad de las generaciones futuras de satisfacer sus propias necesidades (CMMAD,1987).

Según Van Marrewijk (2010), inicialmente el desarrollo sostenible estaba relacionado sólo con temas ambientales; no obstante, en la actualidad incluye los aspectos sociales, ambientales en las actividades empresariales y las interacciones con las partes interesadas. Los modelos, normas, métodos y herramientas en materia de sostenibilidad fueron creados para conseguir la integración de las organizaciones a la sostenibilidad, con orientación a mejorar su impacto social y su reputación. Para Olcese, Rodríguez y Alfaro (2008), las normas son parte de la estrategia de las organizaciones públicas y privadas que evolucionan de acuerdo con sus necesidades.

\subsection{La sostenibilidad empresarial.}

Para sistematizar las prácticas de la sostenibilidad, en las últimas tres décadas, se presentaron diversas soluciones a través de diferentes modelos como acuerdos, convenios y protocolos negociados en diversas cumbres mundiales. A la vez, se instituyeron guías, directrices, normas, protocolos y sistemas de gestión que proporcionan elementos conducentes para debatir los procedimientos que utilizan en las empresas para vigilar la planeación e implementación del desarrollo sostenible (Gil \& Barcellos, 2011). Entonces surge la necesidad de promover el desarrollo económico de modo sostenible como un reto para las organizaciones e instituciones de los gobiernos, por lo que se pusieron en práctica los modelos para el desarrollo sostenible que tienen el propósito de apoyar estos desafíos.

Para Olcese, Rodríguez y Alfaro (2008), las normas en desarrollo sostenible se integran a la dirección estratégica de las empresas. En cuanto a Zdanyte y Neverauskas (2014), consideran que es posible que una organización logre el desarrollo sostenible cuando sus objetivos se establecen en un entorno económico, social y ambiental o en áreas centradas en la implementación de este desarrollo, mediante un cambio gradual de la cultura.

Por otra parte, para Hart y Milstein (2003), el desarrollo sostenible es complicado y multifactorial que no se resuelve con una sola acción empresarial. Así, las organizaciones tienen la meta de prevenir la contaminación disminuyendo sus residuos, empleando tecnologías amigables con el ambiente y limpias. Además, las organizaciones tienen el compromiso de ser socialmente incluyentes con sus partes interesadas mediante interacciones y diálogos, buscarán la manera de desarrollar medidas económicamente factibles para atender los problemas sociales y ambientales que puedan presentarse en el futuro, con una visión de desarrollo sostenible.

De aquí que las organizaciones reaccionen en forma diferente ante el reto que implica el desarrollo sostenible, ya que algunas ignoran los problemas de sostenibilidad; otras actúan sólo en el campo teórico, pero no en su comprensión organizacional y, otros más, intentan completar los aspectos de desarrollo sostenible con las estrategias y conocimientos del negocio. De lo anterior se derivan acciones que son adoptadas por las empresas para lograr el desarrollo sostenible que, en ocasiones, son acompañadas por la aplicación de modelos para su control (Mike, 2013). 
Para analizar los modelos de desarrollo sostenible se necesita precisar información acerca de aquellos modelos actuales, que promueven dicho progreso limpio, que a su vez; son clasificados en normas, políticas, informes e indicadores bursátiles. Dentro de ellos, se pueden caracterizar las dimensiones de sostenibilidad, específicamente en los aspectos de tipo social, ambiental y económico.

Tabla 1. Modelos de desarrollo sostenible.

\begin{tabular}{|c|c|c|c|c|}
\hline Modelos & Año & Autor / lugar & Tipo/Evaluación & Dimensiones \\
\hline Pacto Mundial & $\begin{array}{l}2000,2010 y \\
2017\end{array}$ & $\begin{array}{l}\text { ONU } \\
\text { Suiza }\end{array}$ & Prácticas / Sin evaluación & $\begin{array}{l}\text { Derechos humanos, Condiciones } \\
\text { laborales, Medio ambiente, y } \\
\text { Corrupción }\end{array}$ \\
\hline WCD & 1998, 2000 & $\begin{array}{l}\text { UINC } \\
\text { Suiza }\end{array}$ & $\begin{array}{l}\text { Informe / Evaluación } \\
\text { externa }\end{array}$ & $\begin{array}{l}\text { Social, Ambiental, Económico y } \\
\text { Técnico }\end{array}$ \\
\hline Protocolo IHA & $\begin{array}{l}2004,2006 \\
\text { у } 2012\end{array}$ & $\begin{array}{l}\text { IHA } \\
\text { Reino Unido }\end{array}$ & $\begin{array}{l}\text { Protocolo/Evaluación } \\
\text { externa }\end{array}$ & $\begin{array}{l}\text { Social, Ambiental, Económico y } \\
\text { Técnico }\end{array}$ \\
\hline RSAT & 2010 у 2014 & ECSHDMRC & $\begin{array}{l}\text { Herramienta / Evaluación } \\
\text { externa }\end{array}$ & $\begin{array}{l}\text { Social, Ambiental, Económico y } \\
\text { Técnico }\end{array}$ \\
\hline $\begin{array}{l}\text { Políticas de } \\
\text { seguridad }\end{array}$ & $\begin{array}{l}1997,2006 \\
2012\end{array}$ & $\begin{array}{l}\text { Banco Mundial } \\
\text { Suiza }\end{array}$ & $\begin{array}{l}\text { Políticas / Evaluación } \\
\text { externa }\end{array}$ & Social y Ambiental \\
\hline $\begin{array}{l}\text { Principios de } \\
\text { Ecuador }\end{array}$ & $\begin{array}{l}2003,2006, \\
2013\end{array}$ & $\begin{array}{l}\text { EPFI } \\
\text { Reino Unido }\end{array}$ & $\begin{array}{l}\text { Principios / Evaluación } \\
\text { externa }\end{array}$ & Socia y Ambiental \\
\hline IFC & 2006, 2014 & $\begin{array}{l}\text { Banco Mundial } \\
\text { Suiza }\end{array}$ & $\begin{array}{l}\text { Normas/Evaluación } \\
\text { externa }\end{array}$ & Social y Ambiental \\
\hline BS 8900 & 2006 у 2013 & $\begin{array}{l}\text { BSI } \\
\text { Inglaterra }\end{array}$ & $\begin{array}{l}\text { Estándar/Auditoría } \\
\text { certificable }\end{array}$ & Social, Ambiental y Económico \\
\hline AA 1000 & 1999 у 2008 & $\begin{array}{l}\text { ISEA } \\
\text { Reino Unido }\end{array}$ & $\begin{array}{l}\text { Informe/Auditoría sin } \\
\text { certificar }\end{array}$ & Social (grupos de interés) \\
\hline GRI & $\begin{array}{l}2000,2002 \\
2006 \text { у } 2013\end{array}$ & $\begin{array}{l}\text { GRI } \\
\text { Holanda }\end{array}$ & $\begin{array}{l}\text { Informe/Auditoría } \\
\text { certificable }\end{array}$ & Social, Ambiental y Económico \\
\hline DJSI & 1999 y 2014 & $\begin{array}{l}\text { RobecoSAM's } \\
\text { Estados Unidos }\end{array}$ & $\begin{array}{l}\text { Índices/Verificación } \\
\text { documental }\end{array}$ & Social, Ambiental y Económico \\
\hline FTSE4 Good & 2001 у 2011 & $\begin{array}{l}\text { UNICEF, EIRIS } \\
\text { Reino Unido }\end{array}$ & $\begin{array}{l}\text { Índices/Verificación } \\
\text { documental }\end{array}$ & $\begin{array}{l}\text { Ambiental, Responsabilidad } \\
\text { social y Gobierno corporativo }\end{array}$ \\
\hline IPC Sostenible & 2011 у 2014 & $\begin{array}{l}\text { EIRIS } \\
\text { México }\end{array}$ & $\begin{array}{l}\text { Índices/Verificación } \\
\text { documental }\end{array}$ & $\begin{array}{l}\text { Ambiental, Responsabilidad } \\
\text { social y Gobierno corporativo }\end{array}$ \\
\hline
\end{tabular}

Fuente: Elaboración propia.

Se observa, que los modelos están clasificados en siete normas, tres informes y tres indicadores, además, por lo menos tienen una dimensión de sostenibilidad y se agrupan de la siguiente forma:

- Social. Sólo se incluye el modelo AA1000 que se relaciona con los grupos de interés.

- Social - Ambiental. Se basan en un enfoque de financiación de proyectos de infraestructura; la integran los modelos, las políticas de seguridad del Banco Mundial, el Pacto Mundial, los Principios de Ecuador y los estándares de sostenibilidad del IFC.

- Social - Ambiental - Económica. Se basan en un enfoque más holístico y de aplicación para todo tipo de empresas u organizaciones; la integran los modelos Global Reporting Initiativa y la norma BS 8900:2013 Desarrollo Sostenible.

- Social - Ambiental - Económica. Se basan en un enfoque para empresas que cotizan en índices bursátiles sostenibles; la integran los modelos Dow Jones Sustainability Indexes, FTSE4 Good y el IPC Sostenible. 
- Social - Ambiental - Económica. Se basan en un enfoque de sostenibilidad hidroeléctrica; la integran los modelos World Commission on Dams, Protocolo de Evaluación de Sostenibilidad Hidroeléctrica del IHA y The Rapid Basin-Wide Hydropower Sustainability Assessment Tool el IPC Sostenible.

A continuación, se presenta una descripción breve de los modelos que fomentan el desarrollo sostenible con vigencia al año 2019:

- AA1000 AccontAbility. Son normas de acceso libre y proveen los requerimientos para asegurar la sostenibilidad. Describen un conjunto de procesos que una organización puede seguir para contabilizar, administrar y comunicar su desenvolvimiento social y ético, sin precisar o especificar cuál debiera ser ese desenvolvimiento (AA1000AS, 2008).

- Políticas de seguridad del Banco Mundial. Estas políticas son aplicables a todos los que solicitan préstamos del Banco Mundial. Aquí se establecen obligaciones socioambientales que deben cumplir quienes solicitan financiación para un proyecto, lo que se formaliza de acuerdo con los compromisos del Banco Mundial según los criterios establecidos, tales como transparencia y consulta (Banco Mundial, 2012).

- Pacto Mundial. Prácticas voluntarias en las que las organizaciones se obligan a converger sus directrices y procesos en 10 principios asociados en las cuatro disciplinas (derechos humanos, normas de seguridad, ambiente y anticorrupción). Con más de 9,900 compañías, en 161 países, es la iniciativa corporativa más grande (Global Compact, 2019).

- Principios de Ecuador. Fueron desarrollados por bancos del sector privado, liderados por Citigroup, ABN AMRO, Barclays y WestLB, proporcionan préstamos para proyectos donde el prestatario cumpla con las políticas y procedimientos sociales y ambientales (Principios de Ecuador, 2013).

- The International Finance Corporation (IFC por sus siglas en inglés). Son estándares de desempeño para la gestión de riesgos y la mitigación de impactos ambientales y sociales, incrementando las oportunidades de financiación para proyectos del sector privado de sus países miembros (IFC, 2019).

- Dow Jones Sustainability Indexes (DJSI por sus siglas en inglés). Fue la primera comparación global de sostenibilidad que evaluaba el desempeño sostenible de las 2,500 empresas que figuraban en el Índice del Mercado Global Dow Jones. Los parámetros de este Índice han prevalecido en el mundo durante más tiempo como el punto clave de referencia para la inversión de sostenibilidad en las empresas y para los inversores que toman en cuenta la sostenibilidad al momento de invertir, como en una red de empresas que adoptan las mejores prácticas (RobecoSAM's, 2014).

- FTSE4 Good. Es un índice de referencia con reconocimiento mundial, para inversores que desean identificar empresas con prácticas de sostenibilidad y políticas de responsabilidad social. Los criterios del FTSE4Good se desarrollan a partir de un proceso de consulta al mercado y se aprueban por un comité independiente de expertos (FTSE, s.f.).

- IPC Sostenible. Es un índice de la Bolsa Mexicana de Valores, que agrupa a sus empresas destacadas en materia de sostenibilidad. Los criterios que evalúa son: medio ambiente, responsabilidad social y gobierno corporativo (BMV, 2013).

- The Global Reporting Initiative (GRI por sus siglas en inglés). Es una iniciativa que contiene una guía para desarrollar informes de sostenibilidad con base en las tres esferas clásicas: ambiente, economía y sociedad. Los informes resultantes de las evaluaciones pueden ser publicados en su página (GRI, 2019). 
- Norma BS 8900:2013. Es un estándar para medir la sostenibilidad, se utiliza para que las empresas integren la gestión del desarrollo sostenible en la toma de decisiones que realizan diariamente. Las empresas, sin importar el tamaño o sector, pueden ser auditadas en la gestión del desarrollo sostenible. Esta evaluación está dirigida a los consultores y gerentes responsables de la sostenibilidad en la organización. Además, incluye la rendición de cuentas y la mejora continua (BSI, 2013).

- World Commission on Dams (WCD por sus siglas en inglés). En 1997, se formó WCD patrocinado por el Banco Mundial y ONG ambientales, con los objetivos de revisar la eficacia de las grandes presas y proponer criterios para la planeación, diseño, operación y desmantelamiento. En el 2000, la WCD elaboró su informe final, reconocido como una contribución en el debate sobre cuestiones polémicas derivadas de las grandes presas. Este informe proporcionó un marco adecuado para la toma de decisiones y sustentó el desarrollo de los proyectos de infraestructura que se debían hacer con una evaluación de riesgo y respetar los derechos de los actores durante el proceso (WCD, 2000). A pesar de que este informe suscitó varias discusiones también equilibró los costos y beneficios de las distintas partes interesadas en el proceso, ya que la WCD es una de los principales modelos utilizados para evaluar la sostenibilidad en las grandes presas (PNUMA, s.f.).

- Protocolo de Evaluación de Sostenibilidad Hidroeléctrica. The International Hydropower Association (IHA por sus siglas en inglés) diseñó el protocolo para evaluar la sostenibilidad, la construcción y operación de centrales hidroeléctricas. Así permite la producción de un perfil de sostenibilidad que incluye un sistema de evaluación de proyectos hidroeléctricos a través de la evaluación de buenas prácticas (IHA, 2010).

- The Rapid Basin-Wide Hydropower Sustainability Assessment Tool (RSAT por sus siglas en inglés). Es un modelo de diálogo que reúne a los principales actores de una cuenca fluvial para realizar un análisis estructurado de los temas de sostenibilidad (MRC, s.f.). El RSAT fue diseñado para replicar el enfoque estructurado y completo del Protocolo del IHA en un espectro más graduado para promover la mejora continua y que sirva como una herramienta complementaria del protocolo (RSAT, 2013).

Los modelos presentados permiten identificar las dimensiones de sostenibilidad que los conforman, el tipo de modelo, la evaluación a la que son sujetos, la organización que los emitió, el lugar de publicación, así como el año de sus revisiones.

\subsection{Pregunta de investigación.}

A partir de la descripción y análisis de los modelos de desarrollo sostenible, se propuso confrontar los diversos modelos de desarrollo sostenible, al buscar y establecer criterios que permitan compararlos desde un punto de vista integrado.

A partir de esta perspectiva se concibió la pregunta de investigación: ¿Qué modelo de desarrollo sostenible seleccionar para evaluar la sostenibilidad hidroeléctrica? Además de contar con un método de soporte para la toma de decisiones y lograr jerarquizar los modelos, de un modo práctico y óptimo, con base en criterios de sostenibilidad.

Para dar respuesta al interrogante, a continuación, se muestra el marco teórico, basado en el método proceso analítico jerárquico (AHP); posteriormente, se aplican el método y la recolección de datos conducentes a la toma de decisiones para seleccionar el mejor modelo de desarrollo sostenible, que permita evaluar la sostenibilidad hidroeléctrica; por último, se informan los resultados logrados y sus conclusiones. 


\section{Marco Teórico.}

Las dificultades de la toma de decisiones fueron afrontadas inicialmente de manera monocriterio. Sin embargo, se ha cambiado el protagonismo en beneficio de los métodos multicriterio, donde se tienen varios criterios que frecuentemente entran en conflicto entre sí. Al mismo tiempo, muestran las predilecciones del tomador de decisiones o del grupo responsable de tomar la decisión durante el proceso de selección (Caballero \& Romero, 2006).

\subsection{Proceso Analítico Jerárquico (AHP).}

Para afrontar problemas con múltiples criterios y una cantidad determinada de alternativas, Saaty (1980) presentó el AHP, el proceso de toma de decisiones multicriterio que logra descomponer y ordenar un problema de manera esquemática en una jerarquía de prioridades. Mediante comparaciones pareadas, se instauran jerarquías y se influye en las partes que componen el problema, desplegando reflexiones de valor que discrepan, utilizando la escala de Saaty (ver Tabla 2), para criterios cuantitativos y cualitativos o ambos (Saaty, 1980). La escala de Satty se utiliza para plasmar las comparaciones pareadas que reflejan los pensamientos, los juicios y las intuiciones de los participantes (Casañ, 2013).

Tabla 2. Escala de Saaty.

\begin{tabular}{|c|c|c|}
\hline $\begin{array}{l}\text { Calificación } \\
\text { numérica }\end{array}$ & $\begin{array}{l}\text { Escala verbal de la } \\
\text { preferencia }\end{array}$ & Definición \\
\hline 1 & Igual & Ambos elementos son de igual importancia. \\
\hline 3 & Moderada & Moderada importancia de un elemento sobre otro. \\
\hline 5 & Fuerte & Importancia fuerte de un elemento sobre otro \\
\hline 7 & Muy Fuerte & Importancia demostrada de un elemento sobre otro. \\
\hline 9 & Extrema & Importancia absoluta de un elemento sobre otro \\
\hline $2,4,6,8$ & Términos medios & $\begin{array}{l}\text { Valores intermedios, que se emplean para expresar preferencias } \\
\text { que se encuentran entre dos de las anteriormente indicadas. }\end{array}$ \\
\hline
\end{tabular}

Fuente: Saaty (1997).

Para determinar el autovector, se multiplica al cuadrado la matriz A, a continuación, se normalizan las sumas de las filas y se consigue el valor del autovector. De la Ecuación 1 se despeja $\lambda$ max para calcular el autovalor:

$$
A \cdot x=\lambda \max \cdot x
$$

donde:

A: matriz recíproca

$\mathrm{X}$ : vector propio

$\lambda$ max: máximo valor propio

Para eludir que la decisión se obtenga de juicios con consistencia bajas y sean considerados aleatorios. El método calcula la inconsistencia total de los juicios mediante la proporción de 
consistencia, su porcentaje no deberá ser mayor al diez por ciento. Esto podrá ser influenciado por el tamaño de la matriz A. El índice de consistencia se determina con la Ecuación 2:

$$
C I=(\lambda \max -n) /(n-1)
$$

donde:

$\mathrm{n}$ : tamaño de la matriz

CI: Índice de Consistencia

$\lambda$ max: máximo valor

En último lugar, la razón de consistencia (CR por sus siglas en inglés), se define de tal manera que los valores que estén por encima del 10\% significan inconsistencias en la emisión de los juicios y deberán calcularse reiteradamente hasta conseguir coherencias y consistencias de los mismos. El procesamiento de la información se elabora con base en la Tabla 3.

Tabla 3. Índice de Consistencia.

\begin{tabular}{|c|c|c|c|c|c|c|c|c|c|c|c|}
\hline Tamaño de la matriz (n) & 1 & 2 & 3 & 4 & 5 & 6 & 7 & 8 & 9 & 10 & 11 \\
\hline Consistencia Aleatoria & 0.00 & 0.00 & 0.52 & 0.89 & 1.11 & 1.25 & 1.35 & 1.40 & 1.45 & 1.49 & 1.51 \\
\hline
\end{tabular}

Fuente: Saaty (1997).

Para calcular la razón de consistencia CR, se emplean los índices de consistencia y consistencia aleatorio, la razón de consistencia se determina con la Ecuación 3 (Maurtua, 2006; Márquez, 1999; Ávila, 2000; Castillo et al., 2018):

$$
C R=\frac{C I}{I A}
$$

donde:

CR: Razón de Consistencia

CI: Índice de Consistencia

IA: Índice de Consistencia Aleatoria

Si el resultado de CR es mayor a 0.10 representa que los juicios de la matriz son inconsistentes, y no son aceptables para tomar de decisiones. Para valores de CR iguales o menores a 0.10 las consistencias son aceptables, válidas y justificadas para la toma de decisiones.

El método AHP puede ser utilizado para tomar decisiones en grupo, ya que permite adoptar situaciones de bienestar que no comprometen a la alta dirección de una organización; fortaleciendo las negociaciones y el aprendizaje cognitivo de la organización (Moreno \&Vargas, 2018).

\subsubsection{Etapas del Proceso Analítico Jerárquico (AHP)}

Mediante la representación gráfica de las prioridades del método AHP (Figura 1), se consigue de una manera eficiente y esquemática ordenar los elementos de una problemática compleja decisión, logrando fraccionar las partes que lo integran. 
Figura 1. Etapas del AHP.

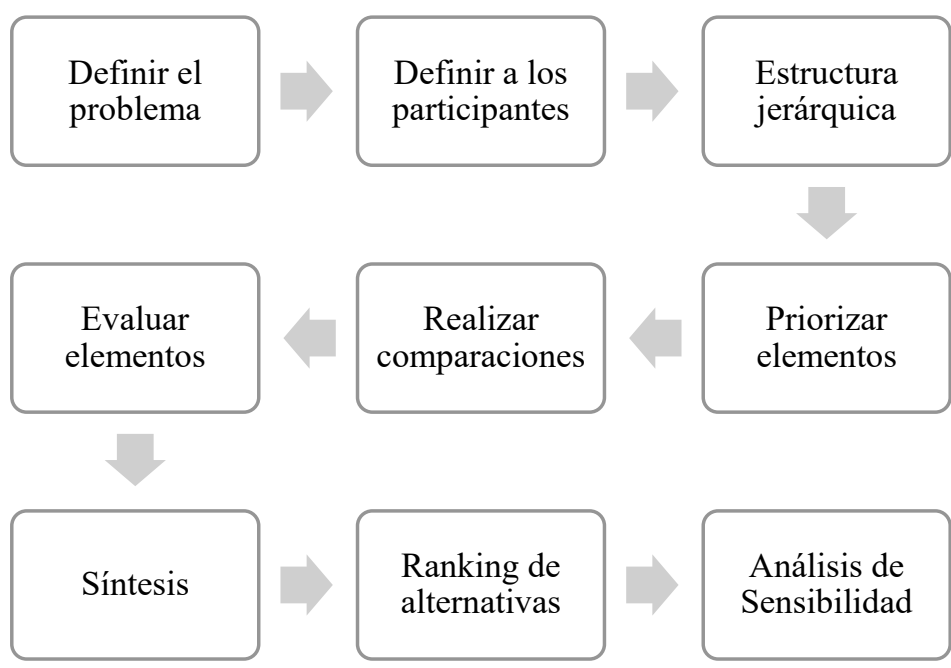

Fuente: Gómez, Soto \& Garduño (2019).

Para Arancibia et al. (2003), Saaty y Vargas (2012), González et al. (2014), el AHP establece las siguientes etapas:

- Definir el problema: se indica el objetivo de la toma de decisiones de manera grupal.

- Definir a los participantes: los participantes deberán ser seleccionados escrupulosamente, ya que en esto radica la representatividad en el método.

- Estructura jerárquica: construir el árbol de decisiones.

- Priorizar elementos: con la información conseguida o compilada de los participantes se combinan los juicios para cada comparación.

- Realizar comparaciones: se inicia con los criterios, se compara su importancia global con el objetivo, después continúa la importancia local de los subcriterios.

- Evaluar elementos: las alternativas se evalúan con cada uno de los subcriterios. Después, se determinan las prioridades locales y globales de las alternativas. Posteriormente, se ejecuta la ponderación final que da cumplimiento al objetivo planteado.

- Síntesis: mediante las comparaciones y la transitividad se logra establecer las jerarquías de las alternativas.

- Ranking de alternativas: se selecciona la mejor alternativa.

- Análisis de Sensibilidad: permite comparar la sensibilidad del resultado con respecto a los posibles cambios en las prioridades de los criterios.

\subsection{Aplicación del método AHP en sostenibilidad energética.}

En trabajos recientes se localizaron referencias que emplean el método AHP en la sostenibilidad energética y energías renovables (Tabla 4).

Algunos de los trabajos emplearon los métodos multicriterio para establecer la comparación de fuentes de energía convencionales versus energías renovables para producir electricidad (Meixner 2009; Ren et al., 2016). El estudio se refiere a la comparación de las energías renovables mediante la 
programación lineal (Afgan \& Carvalho, 2002). Otros trabajos centran su análisis en una perspectiva que les permita establecer criterios de evaluación de la sostenibilidad energética (Heo et al., 2010; Wang et al., 2016; Kocaoglu et al., 2016; Patole et al., 2017; Ishfaq, Ali \& Ali, 2018). Otras publicaciones reflexionan sobre la aplicación de diversos métodos multicriterio en temas energéticos (Escobar \& Moreno, 1994; San Cristóbal, 2012; Al Garni \& Awasthi, 2017; Sagbansua \& Balo, 2017; Azizkhani et al., 2017; Ali et al., 2018; De Prada et al., 2018). Otros estudios sobre el desarrollo y selección de proyectos hidroeléctricos (Supriyasilp, Pongput \& Boonyasirikul, 2009; Patel \& Rana, 2018; Arnaiz et al., 2018; Romanelli et al., 2018). En el trabajo de Strantzali y Aravossis (2016) presenta la clasificación de 183 estudios en materia de energías renovables, publicados de 1983 a 2014.

Tabla 4. Aplicación de métodos multicriterios en sostenibilidad energética.

\begin{tabular}{|c|c|}
\hline Autor & Título de la publicación \\
\hline Afgan \& Carvalho (2002) & Multi-criteria assessment of new and renewable energy power plants \\
\hline Meixner (2009) & $\begin{array}{l}\text { Fuzzy AHP group decision analysis and its application for the evaluation of } \\
\text { energy sources }\end{array}$ \\
\hline $\begin{array}{l}\text { Supriyasilp, Pongput y } \\
\text { Boonyasirikul (2009) }\end{array}$ & Hydropower development priority using MCDM method \\
\hline Heo et al. (2010) & $\begin{array}{l}\text { Analysis of the assessment factors for renewable energy dissemination } \\
\text { program evaluation using fuzzy AHP }\end{array}$ \\
\hline Kaya \& Kahraman (2010) & $\begin{array}{l}\text { Multicriteria renewable energy planning using an integrated fuzzy VIKOR \& } \\
\text { AHP methodology: The case of Istanbul }\end{array}$ \\
\hline San Cristobal (2012) & Multi criteria analysis in the renewable energy industry \\
\hline $\begin{array}{l}\text { Strantzali \& Aravossis } \\
(2016)\end{array}$ & Decision making in renewable energy investments: A review \\
\hline Sánchez et al. (2016) & $\begin{array}{l}\text { Obtaining the Decision Criteria and Evaluation of Optimal Sites for } \\
\text { Renewable Energy Facilities Through a Decision Support System }\end{array}$ \\
\hline Ren et al. (2016) & Sustainability decision support framework for industrial system prioritization \\
\hline Kocaoglu et al. (2016) & $\begin{array}{l}\text { Technology Assessment: Criteria for Evaluating a Sustainable Energy } \\
\text { Portfolio }\end{array}$ \\
\hline Wang et al. (2016) & $\begin{array}{l}\text { A group multi-granularity linguistic-based methodology for prioritizing } \\
\text { engineering characteristics under uncertainties }\end{array}$ \\
\hline Al Garni \& Awasthi (2017) & $\begin{array}{l}\text { Solar PV power plant site selection using a GIS-AHP based approach with } \\
\text { application in Saudi Arabi }\end{array}$ \\
\hline Azizkhani et al. 2017 & $\begin{array}{l}\text { Potential survey of photovoltaic power plants using Analytical Hierarchy } \\
\text { Process (AHP) method in Iran }\end{array}$ \\
\hline Patole et al. (2017) & Energy sector planning using multiple-index pinch analysis \\
\hline Sagbansua \& Balo (2017) & $\begin{array}{l}\text { Decision making model development in increasing wind farm energy } \\
\text { efficiency }\end{array}$ \\
\hline Ali et al. (2018) & $\begin{array}{l}\text { Selection of suitable site in Pakistan for wind power plant installation using } \\
\text { analytic hierarchy process (AHP) }\end{array}$ \\
\hline Ishfa, Ali \& Ali (2018) & $\begin{array}{l}\text { Selection of Optimum Renewable Energy Source for Energy Sector in } \\
\text { Pakistan by Using MCDM Approach }\end{array}$ \\
\hline Patel \& Rana (2018) & $\begin{array}{l}\text { A Selection of the Best Location for a Small Hydro Power Project using the } \\
\text { AHP-Weighted Sum and PROMETHEE Method. }\end{array}$ \\
\hline Arnaiz et al. (2018) & $\begin{array}{l}\text { Facilitating universal energy access for developing countries with micro- } \\
\text { hydropower: Insights from Nepal, Bolivia, Cambodia and the Philippines }\end{array}$ \\
\hline De Prada et al. (2018) & $\begin{array}{l}\text { Territorial Planning: Interactive Multi-Criteria Decision for Urban Patterns. } \\
\text { Case Study: Río Cuarto, Córdoba, Argentina }\end{array}$ \\
\hline Romanelli et al. (2018) & $\begin{array}{l}\text { Site Selection for Hydropower Development: A GIS-Based Framework to } \\
\text { Improve Planning in Brazil. }\end{array}$ \\
\hline
\end{tabular}

Fuente: Elaboración propia. 
En la Tabla 5 se muestra la aplicación del AHP con modelos de desarrollo sostenible.

Tabla 5. Aplicación de métodos multicriterios mediante modelos de sostenibilidad.

\begin{tabular}{ll}
\hline \multicolumn{1}{c}{ Autor } & \multicolumn{1}{c}{ Título de la publicación } \\
\hline Chowdhury et al. (2012) & $\begin{array}{l}\text { An AHP-QFD integrated approach for mitigating barriers of corporate } \\
\text { sustainability. } \\
\text { Corporate sustainability performance assessment: an analytical hierarchy process } \\
\text { approach. }\end{array}$ \\
Goyal \& Rahman (2014) & $\begin{array}{l}\text { Evaluation of Banks' Sustainability Performance in Turkey with Grey Relational } \\
\text { Analysis. }\end{array}$ \\
Özçelik \& Öztürk (2014) & $\begin{array}{l}\text { A fuzzy approach to improve CSR reporting: an application to the Global } \\
\text { Reporting Initiative indicators. }\end{array}$ \\
(2014) & $\begin{array}{l}\text { Sustainable global supplier selection extended towards sustainability risks from } \\
\text { (1+ n) th tier suppliers using fuzzy AHP based approach. }\end{array}$ \\
Gold \& Awashi (2015) & $\begin{array}{l}\text { A fuzzy Analytic Hierarchy Process method to support materiality assessment in } \\
\text { sustainability reporting. }\end{array}$ \\
Calabrese et al. (2016) & $\begin{array}{l}\text { Evaluation of the Three Gorges Dam project using multi-criteria analysis (MCA) } \\
\text { based on a sustainable perspective }\end{array}$ \\
Yue et al. (2018) & Sustainability assessment of a micro hydropower plant in Nepal \\
Bhandari, Saptalena \& & Methodology to assess the market value of companies according to their financial \\
Kusch (2018) & $\begin{array}{l}\text { and social responsibility aspects: An AHP approach } \\
\text { Determination of the Weightings of Hydroelectric Sustainability Criteria by } \\
\text { Combining AHP and GP extended Methods }\end{array}$ \\
\hline
\end{tabular}
Fuente: Elaboración propia.

Algunos trabajos emplearon el AHP para mitigar las barreras de sostenibilidad en las empresas (Menichini \& Rosati 2014; Calabrese, et al., 2016). Los estudios de caso emplean los indicadores del GRI para evaluar la sostenibilidad (Goyal \& Rahman, 2014; Özçelik \& Öztürk, 2014; Gold \& Awashi, 2015; Gómez et al., 2018). Otros estudios emplearon como criterios los temas de sostenibilidad hidroeléctrica del IHA (Yue et al., 2018; Bhandari, Saptalena \& Kusch, 2018; Gómez et al., 2019).

En la revisión de los trabajos anteriores, se identificó que no existe una aplicación directa del método AHP u otro método multicriterio que compare los diversos modelos de desarrollo sostenible, de aquí la importancia de realizar la comparación de los modelos de sostenibilidad y aportar una nueva óptica que facilite su selección conforme a un objetivo predeterminado.

Después de describir la problemática, así como del método AHP, a continuación, se presenta su aplicación, con el objeto de seleccionar el mejor modelo para evaluar la sostenibilidad hidroeléctrica.

\section{Materiales y métodos.}

El presente trabajo espera contribuir a los tomadores de decisiones que buscan comparar los modelos de desarrollo sostenible para realizar una selección con un fin determinado. En el trabajo de campo se utilizó un grupo de enfoque (focus group) para establecer las ponderaciones de los criterios, subcriterios y alternativas del método AHP (Figura 2). 
Figura 2. Árbol de jerarquías para modelos de desarrollo sostenible.

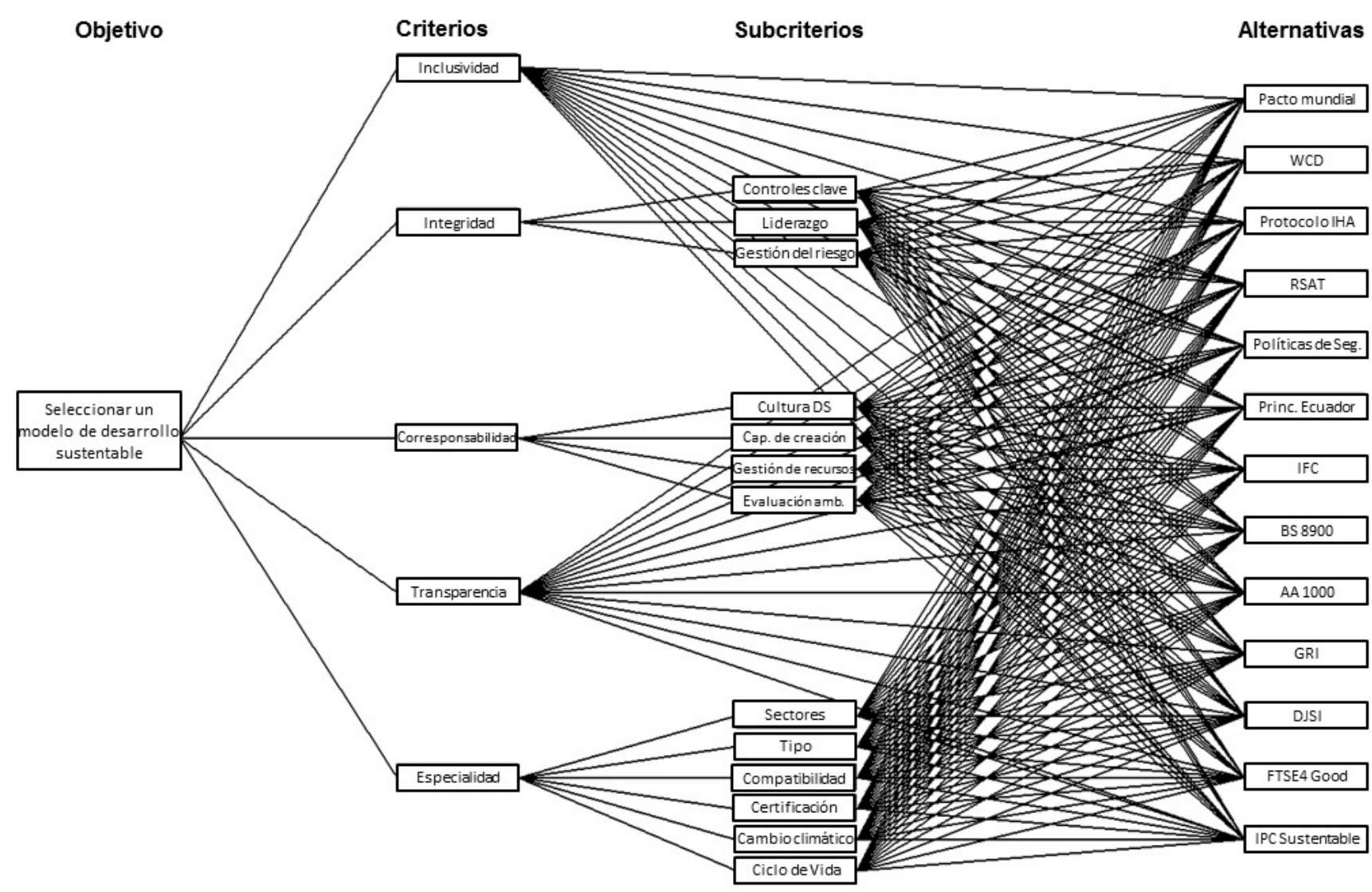

Fuente: Elaboración propia partiendo de la norma BS 8900-1:2013.

Para comparar los modelos de desarrollo sostenible, los criterios seleccionados se establecieron con la norma BS:8900-1:2013 (BSI, 2013):

- Inclusividad: es la intención claramente expresada, o la política de inclusión, de las partes interesadas en el desarrollo de la estrategia de la organización, su planeación y dirección empresarial.

- Integridad: es la adhesión a un conjunto de normas éticas comúnmente aceptadas y comportamientos respetuosos de la legislación.

- Corresponsabilidad: es una posición de responsabilidad que puede ser compartida o es propiedad, en su totalidad, de una persona, comunidad u organización.

- Transparencia: es un principio relacionado con las actividades y las decisiones que perturban a la economía, la sociedad y el ambiente, así como con la voluntad de comunicarlos de una manera clara, precisa, oportuna, veraz y completa.

- Especialidad: son las características particulares que distinguen a los diferentes modelos de desarrollo sostenible.

En este caso, se identificaron 14 subcriterios para inclusividad, integridad, corresponsabilidad y transparencia derivados de la matriz de madurez de desarrollo sostenible (BSI, 2013). Para el criterio de especialidad y sus subcriterios se consideraron las características específicas de cada uno de modelos 
de desarrollo sostenible. Las alternativas identificadas son los modelos de desarrollo sostenible de la Tabla 1.

\subsection{Recolección de datos.}

El Focus Group (grupo de enfoque) se utilizó para obtener las matrices de comparaciones pareadas, solicitadas por el método multicriterio AHP, lo que permitió establecer la prioridad de cada uno de los criterios y subcriterios del árbol de jerarquías. El grupo de enfoque se considera como un tipo de entrevista grupal, que reside en reuniones de grupos pequeños (entre 3 a 10 personas); los participantes conversan en base a uno o varias temáticas expuestas (Hernández et al., 2010).

\subsection{Colectivo a entrevistar.}

En el método AHP lo principal es la experiencia y los conocimientos del grupo entrevistado, más que el número de participantes a entrevistar. El método estudia a expertos concernientes al objetivo del estudio. Las investigaciones cuantitativas admiten el muestro por conveniencia (Schwab, 1995), tomando en cuenta que se deben analizar a los elementos más significativos y ventajosos para la investigación (Grande \& Abascal, 2015). En el mes de febrero de 2019, se realizaron tres reuniones con el grupo de enfoque, con una duración media de 2 horas cada una. Debido a la complejidad de los modelos de desarrollo sostenible, se consideró oportuno que el grupo de expertos cubriera áreas de energía y desarrollo sostenible.

Una muestra no probabilística no requiere representar a los miembros de una población, sino ser una elección de elementos o individuos con características particulares referentes a la problemática (Hernández et al., 2010).

El grupo de enfoque fue conformado por profesores y estudiantes en desarrollo sostenible o temas de energía; ocasionando que se tuviera una variedad de opiniones y punto de vista que difieren sobre la jerarquía de cada uno de los temas y subtemas analizados. Dos de los ocho expertos tienen estudios de licenciatura, tres de maestría y tres cuentan con doctorado. Su experiencia en desarrollo sostenible o energía oscila entre los 5.05 y 20.37 años, con promedio de experiencia de 12.02 años. El 75 por ciento de éstos son mujeres y el 25 por ciento son hombres.

De este modo, el grupo quedó integrado por especialidad:

- Desarrollo sostenible: 5.

- Energía: 3.

\section{Resultados.}

El análisis de resultados aborda los siguientes aspectos:

- El grupo de enfoque determinó las prioridades de los criterios y subcriterios.

- Compararon los modelos de desarrollo sostenible con cada uno de los subcriterios.

Basándose en la escala de Saaty (ver Tabla 2), se procedió a realizar las comparaciones binarias, mediante una matriz de criterios en relación al objetivo específico y subcriterios versus subcriterios respecto a su criterio, estableciendo un valor según su jerarquía respecto al objetivo; además se obtienen las prioridades particulares y generales de cada criterio y subcriterios. 


\subsection{Aplicación del modelo de priorización de modelos de desarrollo sostenible.}

Una vez identificados los criterios de los modelos de desarrollo sostenible, se llega a la evaluación de cada una de los modelos de desarrollo sostenible de acuerdo con los cinco criterios considerados y relacionados con la Tabla 6 , donde se esboza la comparación paritaria entre los criterios considerados, derivando en los resultados siguientes de la comparación.

Tabla 6. Comparación entre criterios de desarrollo sostenible.

\begin{tabular}{|c|c|c|c|c|c|c|}
\hline & 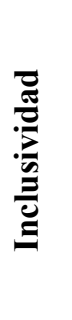 & 胥 & 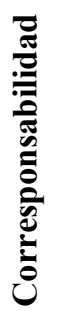 & 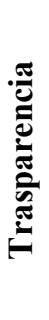 & 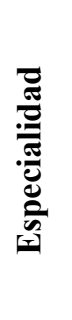 & 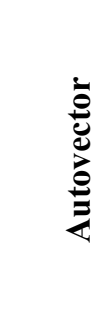 \\
\hline Inclusividad & 1 & $1 / 2$ & $1 / 4$ & 2 & $1 / 6$ & 0.078 \\
\hline Integridad & 2 & 1 & $1 / 2$ & 3 & $1 / 4$ & 0.138 \\
\hline Corresponsabilidad & 4 & 2 & 1 & 5 & $1 / 2$ & 0.265 \\
\hline Trasparencia & $1 / 2$ & $1 / 3$ & $1 / 5$ & 1 & $1 / 7$ & 0.051 \\
\hline Especialidad & 6 & 4 & 2 & 7 & 1 & 0.469 \\
\hline
\end{tabular}

Fuente: Elaboración propia.

Los resultados derivados de la Tabla 6 reportan que el criterio con mejor posición es el de Especialidad, ya que obtuvo una prioridad de $46.9 \%$ ciento. El siguiente fue el criterio de Corresponsabilidad, con una prioridad de $26.2 \%$. Finalmente, en la última posición se ubicó el criterio de Trasparencia, con una prioridad de $5.1 \%$.

El cálculo de $\lambda \max$ se obtiene mediante el promedio de los valores de la matriz $A \cdot x$ entre $x$ :

$$
\lambda_{\max }=\frac{A \cdot x}{x}=\text { Promedio }\left\{\frac{0.392}{0.078}, \frac{0.694}{0.138}, \frac{1.338}{0.265}, \frac{0.255}{0.051}, \frac{2.369}{0.469}\right\}=5.049
$$

A continuación, se calcula el índice de consistencia:

$$
C I=\frac{\lambda_{\max }-n}{n-1}=\frac{5.049-5}{5-1}=0.012
$$

Posteriormente, se obtiene la razón de consistencia, tomando el valor de IA de la Tabla 3.

$$
C R=\frac{C I}{I A}=\frac{0.012}{1.11}=0.0109 \leq 0.10
$$

El resumen de los resultados anteriores, se observan en la Tabla 7. Los resultados obtenidos en la Tabla 7 señalan que la razón de consistencia CR, es de 0.0109 o del $1.09 \%$ por lo que está por debajo del $10 \%$ y se considera consistente los valores de las comparaciones de la matriz de la Tabla 6. 
Tabla 7. Determinación del autovalor de la matriz A.

\begin{tabular}{ccccc}
\hline Autovector & $A \cdot x$ & $\lambda=\frac{A \cdot x}{x} \quad C I=\frac{\lambda_{\max }-n}{n-1}$ & $C R=\frac{C I}{I A}$ \\
\hline 0.078 & 0.392 & 5.049 & \\
0.138 & 0.694 & 5.049 & 0.0109 \\
0.265 & 1.338 & 5.049 & 0.012 \\
0.051 & 0.255 & 5.049 & \\
0.469 & 2.369 & 5.049 & \\
\hline \multicolumn{5}{c}{ Fuente: Elaboración propia. }
\end{tabular}

De esta misma forma se obtuvieron los subcriterios de integridad, corresponsabilidad y especialidad, que se presentan en las Tablas 8, 9 y 10; en el resumen de la Figura 3 se observan las ponderaciones globales y locales.

Tabla 8. Subcriterios de integridad.

\begin{tabular}{lcccc}
\hline & Controles clave & Liderazgo & Gestión del riesgo & Autovector \\
\hline Controles clave & 1 & $1 / 2$ & 2 & 0.297 \\
Liderazgo & 2 & 1 & 3 & 0.540 \\
Gestión del riesgo & $1 / 2$ & $1 / 3$ & 1 & 0.163 \\
\hline $\mathrm{CR}=0.061$ & &
\end{tabular}

Fuente: Elaboración propia.

Tabla 9. Subcriterios de corresponsabilidad.

\begin{tabular}{|c|c|c|c|c|c|}
\hline & 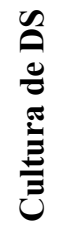 & 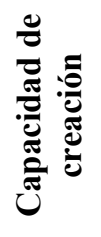 & 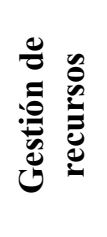 & 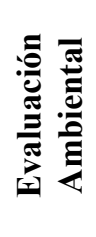 & 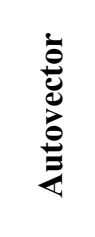 \\
\hline Cultura de DS & 1 & 2 & $1 / 2$ & $1 / 3$ & 0.157 \\
\hline Capacidad de creación & $1 / 2$ & 1 & $1 / 3$ & $1 / 5$ & 0.088 \\
\hline Gestión de recursos & 2 & 3 & 1 & $1 / 2$ & 0.272 \\
\hline Evaluación Ambiental & 3 & 5 & 2 & 1 & 0.483 \\
\hline
\end{tabular}

$\mathrm{CR}=0.066$

Fuente: Elaboración propia. 
Tabla 10. Subcriterios de especialidad.

\begin{tabular}{|c|c|c|c|c|c|c|c|}
\hline & 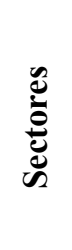 & 气 & 苞 & $\begin{array}{l}\frac{\pi}{0} \\
\vdots \\
0 \\
0 \\
0 \\
0 \\
0\end{array}$ & 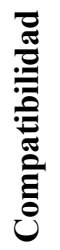 & 刍 & 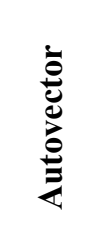 \\
\hline Sectores & 1 & 5 & 2 & 3 & 7 & 3 & 0.386 \\
\hline Tipo & $1 / 5$ & 1 & $1 / 3$ & $1 / 2$ & 2 & $1 / 2$ & 0.075 \\
\hline Certificación o verificación & $1 / 2$ & 3 & 1 & 2 & 5 & 2 & 0.232 \\
\hline Ciclo de Vida & $1 / 3$ & 2 & $1 / 2$ & 1 & 3 & 1 & 0.131 \\
\hline Compatibilidad & $1 / 7$ & $1 / 2$ & $1 / 5$ & $1 / 3$ & 1 & $1 / 3$ & 0.045 \\
\hline Cambio climático & $1 / 3$ & 2 & $1 / 2$ & 1 & 3 & 1 & 0.31 \\
\hline
\end{tabular}

Fuente: Elaboración propia.

Figura 3. Ponderaciones de componentes del modelo jerárquico.

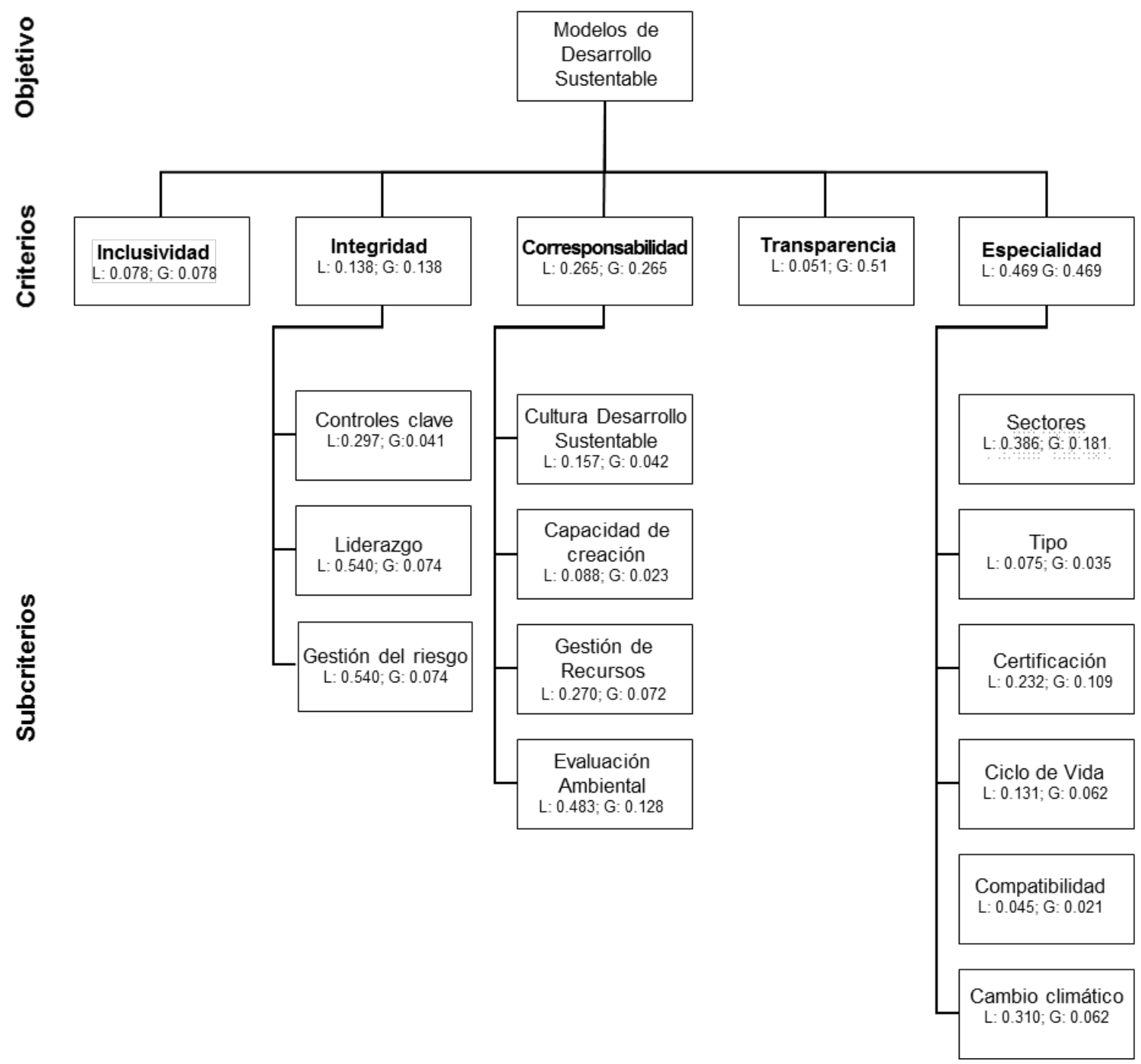

Fuente: Elaboración propia. 


\subsection{Jerarquización de alternativas y síntesis.}

Con la totalidad de comparaciones, se obtiene el ordenamiento de las alternativas, gracias a la jerarquización de estos modelos. La síntesis de los subcriterios en relación a su criterio con las ponderaciones totales, se efectuó en la última comparación en relación al objetivo establecido (Tabla $11)$.

Tabla 11. Comparaciones entre los modelos de desarrollo sostenible.

\begin{tabular}{ccccccc}
\hline \multirow{2}{*}{ Alternativas } & \multicolumn{5}{c}{ Criterios } & $\begin{array}{c}\text { Ponderación } \\
\text { de las } \\
\text { Altenativas }\end{array}$ \\
\cline { 2 - 6 } Inclusividad & Integridad & Corresponsabilidad & Trasparencia & Especialidad & 0.0699 \\
AA 1000 & 0.1279 & 0.0658 & 0.0565 & 0.1185 & 0.0640 & 0.0917 \\
GRI & 0.1279 & 0.0827 & 0.1025 & 0.1185 & 0.0793 & 0.0446 \\
IFC & 0.0387 & 0.0300 & 0.0395 & 0.0360 & 0.0536 & 0.1139 \\
Protocolo IHA & 0.1279 & 0.1244 & 0.1025 & 0.1185 & 0.1145 & 0.1109 \\
BS 8900 & 0.1279 & 0.1244 & 0.1299 & 0.1185 & 0.0926 & 0.1011 \\
DJSI & 0.0672 & 0.1150 & 0.0806 & 0.0628 & 0.1184 & 0.0475 \\
Pacto Mundial & 0.0232 & 0.0347 & 0.0452 & 0.0628 & 0.0549 & 0.0779 \\
FTSE4 Good & 0.0672 & 0.0827 & 0.0933 & 0.0628 & 0.0713 & 0.0681 \\
IPC Sostenibilidad & 0.0672 & 0.0827 & 0.0806 & 0.0628 & 0.0575 & 0.0895 \\
WCD & 0.0672 & 0.1045 & 0.0700 & 0.1185 & 0.0967 & 0.0940 \\
RSAT & 0.0672 & 0.0921 & 0.1010 & 0.0628 & 0.0984 & 0.0359 \\
Política de & 0.0232 & 0.0264 & 0.0307 & 0.0215 & 0.0454 & 0.0549 \\
Seguridad & & & & 0.0360 & 0.0536 & \\
Principios de & 0.0672 & 0.0347 & 0.0678 & $\mathbf{0 . 0 5 0 6}$ & $\mathbf{0 . 4 6 3}$ & \\
Ecuador & & $\mathbf{0 . 0 7 7 6}$ & $\mathbf{0 . 1 3 7 5}$ & $\mathbf{0 . 2 6 5 0}$ & $\mathbf{0 . 0 5 0 6}$ \\
Ponderaciones & $\mathbf{0 . 0 7 5}$
\end{tabular}

Fuente: Elaboración propia.

Con el cálculo valores globales y locales de los criterios y subcriterios, se consiguen los resultados finales y el orden para cada una de las alternativas (Tabla 12).

Tabla 12. Ranking de alternativas.

\begin{tabular}{ccc}
\hline Alternativas & Ponderación Global & Orden \\
\hline Protocolo IHA & 0.1139 & 1 \\
BS 8900 & 0.1109 & 2 \\
DJSI & 0.1011 & 3 \\
GRI & 0.0917 & 4 \\
RSAT & 0.094 & 5 \\
WCD & 0.0895 & 6 \\
FTSE4 Good & 0.0779 & 7 \\
AA 1000 & 0.0699 & 8 \\
IPC Sostenible & 0.0681 & 9 \\
Principios de Ecuador & 0.0549 & 10 \\
Pacto Mundial & 0.0475 & 11 \\
IFC & 0.0446 & 12 \\
Políticas de Seguridad & 0.0359 & 13 \\
\hline
\end{tabular}

Fuente: Elaboración propia. 
Por lo anterior y como resultado del análisis de los modelos comparados, el Protocolo de la IHA es el modelo de desarrollo sostenible más apropiado para evaluar la sostenibilidad hidroeléctrica: como se desprende del análisis y datos reportados, obtuvo una prioridad de $11.4 \%$. El segundo modelo fue el BS 8900 , con una prioridad de $11.1 \%$. Finalmente, las políticas de seguridad son las que se ubican en el último lugar, con una prioridad de $3.65 \%$.

\subsection{Análisis de Sensibilidad.}

A continuación, se presenta un análisis de sensibilidad por escenarios considerando cambios significativos en las ponderaciones de los criterios (Tabla 13):

1. Inclusividad como criterio más importante

2. Integridad como criterio más importante

3. Corresponsabilidad como criterio más importante

4. Transparencia como criterio más importante

Este análisis emplea herramientas informáticas de cálculo (en este caso se empleó el software Web-HIPRE). El análisis consiste en realizar variaciones en las ponderaciones de los criterios y observar, numérica y gráficamente, cómo estos cambios afectan al resto de los valores de las alternativas y su jerarquización (Figura 4).

Es importante tener en cuenta que existen muchas más posibilidades, como las anteriores, para realizar permutaciones y/o alteraciones a los valores de los criterios, que permiten al método ser fluctuante ante posibles adecuaciones por actualizaciones de los modelos de desarrollo sostenible.

En la Tabla 13 se muestran las ponderaciones de los modelos en cada escenario.

Tabla 13. Escenarios del análisis de sensibilidad.

\begin{tabular}{lccccc}
\hline \multirow{2}{*}{ Alternativas } & \multicolumn{5}{c}{ Valores } \\
\cline { 2 - 6 } & Inicial & Escenario 1 & Escenario 2 & Escenario 3 & Escenario 4 \\
\hline AA 1000 & 0.0699 & 0.092 & 0.069 & 0.067 & 0.090 \\
GRI & 0.0917 & 0.106 & 0.089 & 0.094 & 0.103 \\
IFC & 0.0446 & 0.042 & 0.040 & 0.043 & 0.041 \\
Protocolo IHA & 0.1139 & 0.119 & 0.118 & 0.111 & 0.116 \\
BS 8900 & 0.1109 & 0.117 & 0.115 & 0.115 & 0.114 \\
DJSI & 0.1011 & 0.088 & 0.106 & 0.096 & 0.085 \\
Pacto Mundial & 0.0475 & 0.038 & 0.043 & 0.047 & 0.054 \\
FTSE4 Good & 0.0779 & 0.074 & 0.079 & 0.081 & 0.072 \\
IPC Sostenibilidad & 0.0681 & 0.068 & 0.073 & 0.071 & 0.066 \\
WCD & 0.0895 & 0.081 & 0.095 & 0.085 & 0.101 \\
RSAT & 0.0940 & 0.084 & 0.094 & 0.096 & 0.081 \\
Política de Seguridad & 0.0359 & 0.031 & 0.032 & 0.034 & 0.030 \\
Principios de Ecuador & 0.0549 & 0.060 & 0.048 & 0.058 & 0.047 \\
\hline
\end{tabular}

Fuente: Elaboración propia. 
Figura 4. Análisis de sensibilidad del Ranking de alternativas.

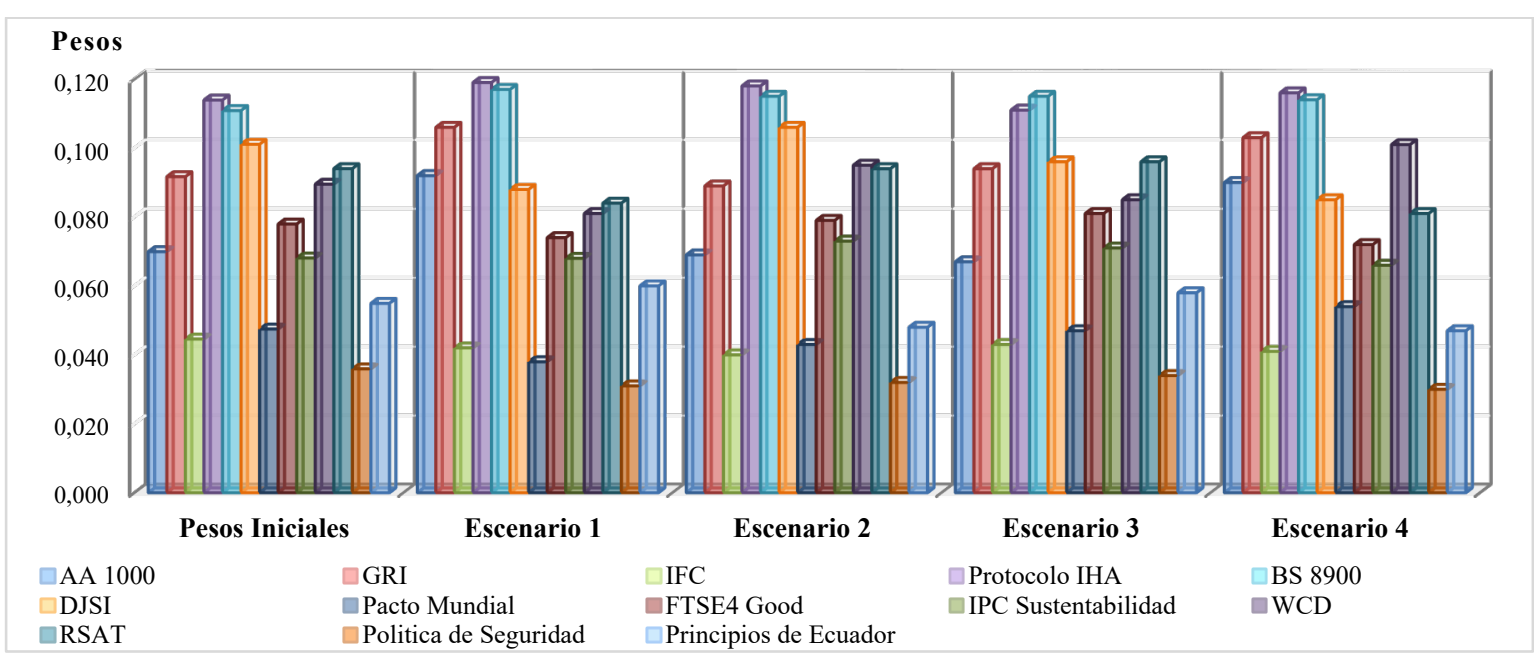

Fuente: Elaboración propia.

Los resultados obtenidos de la Tabla 13 y graficados en la Figura 4, muestran los cuatro escenarios y los cambios en las ponderaciones de los criterios. Sólo en el escenario tres al asignarle un mayor valor al criterio de corresponsabilidad, el modelo de la norma BS 8900 ocupa el primer puesto. En los otros escenarios el Protocolo del IHA se mantiene en el primer sitio y, en todos los escenarios, el modelo de políticas de seguridad del Banco Mundial obtuvo la menor ponderación.

\section{Conclusiones.}

Después de analizar los modelos de desarrollo sostenible, se detalló la aplicación del método multicriterio AHP que se empleó para jerarquizarlos. Aquí se identificaron algunos aciertos, en particular con modelo del protocolo del IHA que permite enunciar los siguientes aspectos conclusivos:

- Con base en el análisis de resultados se determinó que el Protocolo de la IHA es el modelo de desarrollo sostenible más apropiado para evaluar la sostenibilidad hidroeléctrica con un $11.3 \%$. Otros modelos que también evalúan la sostenibilidad hidroeléctrica fueron RSAT y WCD que obtuvieron ponderaciones del $9.4 \%$ y $8.95 \%$ respectivamente.

- En el análisis de sensibilidad empleando el software Web-HIPRE, se observa que de los cuatros escenarios propuestos en tres el Protocolo de la IHA mantiene el primer lugar, validando su selección; sólo en el escenario donde se le asignó mayor valor al criterio de corresponsabilidad, el modelo del BS 8900 ocupa el primer puesto.

- Se observa que el protocolo del IHA se pueden beneficiar de otros modelos, como del BS 8900 al incorporar la sostenibilidad al sistema de gestión de la organización; las normas AA1000 facilitan la rendición de cuentas a las partes interesadas; el GRI fomenta la trasparencia a través de informes con indicadores de sostenibilidad; lo anterior permite robustecer, evidenciar y adoptar las mejores prácticas en el sector hidroeléctrico.

- El trabajo ayuda a quienes buscan justificar su decisión de seleccionar un modelo de desarrollo sostenible, ya que permite incorporar más criterios, subcriterios y alternativas a los indicados en este estudio, por la flexibilidad y simplicidad del método AHP. Por otra parte, cabe la mención que cuanto mayor sea la agregación, el método AHP mostrará mayores complicaciones, lo que ocasionaría desconciertos en los cálculos de su operación. 
- Se cumplió el objetivo que se centró en la caracterización de los modelos mediante la identificación de criterios de sostenibilidad, de donde se eligió el más adecuado, como se evidenció en los elementos conclusivos incluidos, para atender diferentes necesidades como las de la sostenibilidad hidroeléctrica.

Por lo anterior, me permito recomendar con apego a los resultados de este estudio que considero factible la aplicación de este modelo en las disciplinas de calidad, medio ambiente, seguridad y salud en el trabajo, gestión de riesgo, partes interesadas, entre otros. También se podrán incorporar en trabajos futuros otros criterios, subcriterios o modelos de desarrollo sostenible, así como la utilización de otros métodos multicriterio grupales como el AHP-GP y fuzzy opinión.

\section{Referencias}

AccounAbility (2008). AA1000APS: Norma de Principio de Sostenibilidad. London: AccounAbility.

Afgan, N., \& Carvalho, M. (2002). Multi-criteria assessment of new and renewable energy power plants. Energy, 27(8), 739-755 . DOI: 10.1016/S0360-5442(02)00019-1.

Ali, Y., Butt, M., Sabir, M., Mumtaz, U., \& Salman, A. (2018). Selection of suitable site in Pakistan for wind power plant installation using analytic hierarchy process (AHP). Journal of Control and Decision, 5(2), 117-128. DOI: 10.1080/23307706.2017.1346490.

Al Garni, H., \& Awasthi, A. (2017). Solar PV power plant site selection using a GIS-AHP based approach with application in Saudi Arabia. Applied Energy, 206, 1225-1240. DOI: 10.1016/j.apenergy.2017.10.024.

Arancibia, S., Contreras, E., Mella, S., Torres, P., \& Villablanca, I. (2003). Evaluación Multicriterio: aplicación para la formulación de proyectos de infraestructura deportiva. Memoria de Ingeniero Civil Industrial. Universidad de Chile, Santiago.

Arnaiz, M., Cochrane, T., Dudley, N., \& Chang, T. (2018). Facilitating universal energy access for developing countries with micro-hydropower: Insights from Nepal, Bolivia, Cambodia and the Philippines. Energy Research \& Social Science, 46, 356-367. DOI: 10.1016/j.erss.2018.07.016.

Ávila, R. (2000). El AHP, proceso analítico jerárquico y su aplicación para determinar los usos de las tierras: El caso de Brasil. Informe técnico. Proyecto Regional Información sobre Tierras y Aguas para un Desarrollo Agrícola Sostenible (Proyecto Gcp/Rla/126/Jpn). Santiago, Chile.

Azizkhani, M., Vakili, A., Noorollahi, Y., \& Naseri, F. (2017). Potential survey of photovoltaic power plants using Analytical Hierarchy Process (AHP) method in Iran. Renewable and Sustainable Energy Reviews, 75, 1198-1206. DOI: 10.1016/j.rser.2016.11.103BSI (2013).

BS 8900-1:2013 (2013). Managing sustainable development of organizations. Guide. London: The British Standards Institution.

Banco Mundial (2012). Safeguard Policies. Recuperado de http://go.worldbank.org/WTA1ODE7T0.

Bhandari, R., Saptalena, L.G., \& Kusch, W. (2018). Sustainability assessment of a micro hydropower plant in Nepal. Energy, Sustainability and Society, 8(1), 1-15. DOI: 10.1186/s13705-018-0147-2. 
BMV (2013). Guía de ayuda para las Empresas Emisoras que cotizan en la Bolsa Mexicana respecto de la información que deberá estar disponible de manera pública y ser susceptible de evaluación para el Índice IPC Sustentable. México: Bolsa Mexicana de Valores.

BSI (2013). BS 8900-1. Managing sustainable development of organizations. London: The British Standards Institution.

Caballero, R., \& Romero, C. (2006). Teoría de la Decisión Multicriterio: Un Ejemplo de Revolución Científica Kuhniana. Boletín de Estadística e Investigación Operativa, 22 (4), 9-15.

Calabrese, A., Costa, R., Levialdi, N., \& Menichini, T. (2016). A fuzzy analytic hierarchy process method to support materiality assessment in sustainability reporting. Journal of Cleaner Production, 121, 248-264. DOI: 10.1016/j.jclepro.2015.12.005.

Casañ, A. (2013). La desición multicriterio; aplicación en las selección de ofertas competitivas en edificación (Tesis de maestría), Universidad Politecnica de Valencia, Valencia.

Castillo, M., Álvarez, A., Alfaro, M., Sánchez, J., \& Quezada, I. (2018). Factores clave en el desarrollo de la capacidad emprendedora de estudiantes universitarios. Revista de Métodos Cuantitativos para la Economía y la Empresa, (25), 111-129.

CMMAD (1987). Nuestro Futuro Común. Madrid: Alianza Editorial.

De Prada, J., Degioanni, A., Cisneros, J. Cantero, A., Gil, H., Tello, D., .Pereyra, C., \& Giayetto, O. (2018). Planificación territorial: elección multicriterio interactiva del patrón de urbanización. Estudio de caso: Río Cuarto, Córdoba, Argentina. Revista de Métodos Cuantitativos para la Economía y la Empresa, (26), 25-51.

Escobar, M., \& Moreno, J. (1994). Técnicas multicriterio discretas en la planificación de cuencas fluviales. Estudios de Economía Aplicada, (1), 7-30.

FTSE (s.f.). Índice FTSE4Good IBEX: Informe de Investigación y análisis. Madrid: Grupo FTSE.

Gil, A., \& Barcellos, L. (2011). Los desafíos para la sostenibilidad empresarial en el siglo XXI. Revista Galega de Economía, 20 (2), 1-22.

Global Compact (2019). Global Compact. Who we are. Recuperado de https://www.unglobalcompact.org/what-is-gc

Gold, S., \& Awasthi, A. (2015). Sustainable global supplier selection extended towards sustainability risks from $(1+n)$ th tier suppliers using fuzzy AHP based approach. IFAC-PapersOnLine, 48(3), 966-971. DOI: 10.1016/j.ifacol.2015.06.208.

Gómez, T., García, M., Guijarro, F., \& Preuss, M. (2018). Methodology to assess the market value of companies according to their financial and social responsibility aspects: An AHP approach. Journal of the Operational Research Society, 69(10), 1599-1608. DOI: 10.1057/s41274-017-0222-7. 
Gómez, J., Soto, R., \& Garduño, S. (2019). Determinación de las Ponderaciones de los Criterios de Sustentabilidad Hidroeléctrica mediante la Combinación de los Métodos AHP y GP Extendida. Ingeniería, 24(2), 116-142. DOI: 10.14483/23448393.14469.

González, C., Garza, R., \& Pérez, E. (2014). Enfoque híbrido simulación-proceso analítico jerárquico: caso de estudio del rediseño de un restaurante. Revista de Métodos Cuantitativos para la Economía y la Empresa, (17), 23-41.

Goyal, P., \& Rahman, Z. (2014). Corporate sustainability performance assessment: an analytical hierarchy process approach. International Journal of Intercultural Information Management, 4 (1), 1-14. DOI: 10.1504/IJIIM.2014.065285.

Grande, I., \& Abascal, E. (2014). Fundamentos y Técnicas de Investigación Comercial. Madrid: ESIC Editorial.

GRI (2019). Information. About GRI. Recuperado de https://www.globalreporting.org/information/about-gri/Pages/default.aspx

Grisales, E., \& Murillo, J. (2014). El mercado de bonos de carbono y su aplicación para proyectos hidroeléctricos. Revista CINTEX, 18, 131-143.

Hart, S., \& Milstein, M. (2003). Creating Sustainable Value. The Academy of Management Executive, 17(2), 56-67. DOI: 10.5465/AME.2003.10025194.

Heo, E., Kim, J., \& Boo, K. (2010). Analysis of the assessment factors for renewable energy dissemination program evaluation using fuzzy AHP. Renewable and Sustainable Energy Reviews, 14(8), 2214-2220. DOI: 10.1016/j.rser.2010.01.020.

Hernández, R., Fernández, C. \& Baptista, P. (2010). Metodología de la Investigación. México: McGraw-Hill.

IFC (2019). About IFC. Overview. Recuperado de https://www.ifc.org/wps/wcm/connect/corp_ext_content/ifc_external_corporate_site/about + ifc_ne $\mathrm{W}$

IHA (2010). Protocolo de Evaluación de la Sostenibilidad de la Hidroelectricidad. London: Asociación Internacional de la Energía Hidroeléctrica.

Ishfaq, S., Ali, S., \& Ali, Y. (2018). Selection of Optimum Renewable Energy Source for Energy Sector in Pakistan by Using MCDM Approach. Process Integration and Optimization for Sustainability, 2(1), 61-71. DOI: 10.1007/s41660-017-0032-z.

Kocaoglu, D., Daim, T., Iskin, I., \& Alizadeh, Y. (2016). Technology Assessment: Criteria for Evaluating a Sustainable Energy Portfolio. In T. U. Daim (Ed.), Hierarchical Decision Modeling: Essays in Honor of Dundar F. Kocaoglu (pp. 3-34). Cham: Springer International Publishing.

Márquez, H. (1999). Métodos matemáticos de evaluación de factores de riesgo para el Patrimonio Arqueológico: una aplicación Gis del método de jerarquías analíticas de TL Saaty. Spal, 6, 21-37. 
Maurtua, D. (2006). Criterios de Selección de Personal mediante el uso del proceso de análisis jerárquico. Aplicación en la selección de personal para la Empresa Exotic Foods SAC. (Licenciado en Investigación Operativa), Universidad Nacional Mayor de San Marcos, Lima, Perú.

Meixner, O. (2009). Fuzzy AHP group decision analysis and its application for the evaluation of energy sources. In Proceedings of the 10th International Symposium on the Analytic Hierarchy/Network Process, Pittsburgh, PA, USA.

Menichini, T., \& Rosati, F. (2014). A fuzzy approach to improve CSR reporting: an application to the Global Reporting Initiative indicators. Procedia-Social and behavioral Sciences, 109, 355-359. DOI: $10.1016 /$ j.sbspro.2013.12.471.

Mike, J. (2013). Reflections from EGOS 2012: culture, design and sustainability. South Asian Journal of Global Business Research, 2(1), 33-42. DOI: 10.1108/20454451311303275.

Moreno, J., \& Vargas, L. (2018). Decisión Multicriterio Cognitiva y el Legado del Proceso Analítico Jerárquico. Estudios de Economía Aplicada, 36, 67-80.

MRC (s.f.). RSAT Overview: The Basin-wide Hydropower Sustainability Assessment Tool. Mekong River Commission. Recuperado de http://www.mrcmekong.org/about-mrc/programmes/initiativeon-sustainable-hydropower/rsat-overview-the-basin-wide-hydropower-sustainability-assessmenttool/

Olcese, A., Rodríguez, M., \& Alfaro, J. (2008). Manual de la empresa responsable y sostenible: Conceptos y herramientas de la Responsabilidad Social Corporativa o de la Empresa. Madrid: McGraw-Hill.

Özçelik, F., \& Öztürk, B. (2014). Evaluation of Banks' Sustainability Performance in Turkey with Grey Relational Analysis. Muhasebe ve Finansman Dergisi, (63), 189-209.

Patel, J., \& Rana, S. (2018). A Selection of the Best Location for a Small Hydro Power Project using the AHP-Weighted Sum and PROMETHEE Method. Pertanika Journal of Science and Technology, 26(4), 1591-1603.

Patole, M., Bandyopadhyay, S., Foo, D., \& Tan, R. (2017). Energy sector planning using multiple-index pinch analysis. Clean Technologies and Environmental Policy, 19(7), 1967-1975. DOI: 10.1007/s10098-017-1365-6.

PNUMA (s.f.). Dams and development project. United Nations Environment Programmes: The World Commission on Dams.

Principios de Ecuador (2013). Una referencia del sector financiero para determinar, evaluar y gestionar los riesgos ambientales y sociales de los proyectos. Equar Principles.

Quintana, G. (2011). Antecedentes y Marco Conceptual del Desarrollo Sustentable. Villavicencio, M. (Ed.): Desarrollo Sustentable en el contexto actual (pp. 7-48). México. 
Ren, J., Xu, D., Cao, H., Wei, S., Dong, L., \& Goodsite, M. (2016). Sustainability decision support framework for industrial system prioritization. AIChE Journal, 62(1), 108-130. DOI: 10.1002/aic.15039.

RobecoSAM's (2014). Corporate Sustainability Assessment Methodology. Zurinch: RobecoSAM's AG.

Romanelli, J., Silva, L., Horta, A., \& Picoli, R. (2018). Site Selection for Hydropower Development: A GIS-Based Framework to Improve Planning in Brazil. Journal of Environmental Engineering, 144(7), 1-10 . DOI: 10.1061/(ASCE)EE.1943-7870.0001381.

RSAT (2013). Draff The Manual Rapid Basin-wide Hydropower Sustainability Assessment Tool Version 4.

Saaty, T. (1980). Multicriteria decision Making:The Analytic Hierarchy Process. New York: McGraw Hill.

Saaty, T. (1997). Toma de Decisiones para Líderes: El proceso analítico jerárquico la toma de decisiones en un mundo complejo. Pittsburgh: RWS Publications.

Saaty, T. \& Vargas, L. (2012). Models, Methods, Concepts \& Applications of the Analytic Hierarchy Process. Springer.

San Cristóbal, J. (2012). Multi criteria analysis in the renewable energy industry. Springer, London: Springer Science \& Business Media.

Sagbansua, L., \& Balo, F. (2017). Decision making model development in increasing wind farm energy efficiency. Renewable Energy, 109, 354-362. DOI: 10.1016/j.renene.2017.03.045.

Schwab, R. (1995). Twenty years of policy recommendations for indigenous education: overview and research implications. Australian: Australian National University.

Strantzali, E., \& Aravossis, K. (2016). Decision making in renewable energy investments: A review. Renewable and Sustainable Energy Reviews, 55, 885-898. DOI: 10.1016/j.rser.2015.11.021.

Supriyasilp, T., Pongput, K., \& Boonyasirikul, T. (2009). Hydropower development priority using MCDM method. Energy Policy, 37(5), 1866-1875. DOI: 10.1016/j.enpol.2009.01.023.

Van Marrewijk, M. (2010). A Typology of Institutional Frameworks for Organizations. Technology and Investment, 1(02), 101-109. DOI: 10.4236/ti.2010.12012.

Wang, Z., Fung, R., Li, Y., \& Pu, Y. (2016). A group multi-granularity linguistic-based methodology for prioritizing engineering characteristics under uncertainties. Computers \& Industrial Engineering, 91, 178-187. DOI: 10.1016/j.cie.2015.11.012.

WCD (2000). Dams and Development: A New Framework for Decision-Making The Report of the World Commission on Dams. London: World Commission on Dams. 
WEB-Hipre: Global decision support (Version 1.22) [software de computación] (2007). Helsinki University of Technology: Systems Analysis Laboratory.

Yue, H., Wei, Z., Junshan, G., Yihe, M., Junqi, D., Lingkai, Z., \& Yanpeng, Z. (2018). Evaluation of the Three Gorges Dam project using multi-criteria analysis (MCA) based on a sustainable perspective. IOP Conference Series: Earth and Environmental Science, 121(5), 1-7. DOI:10.1088/1755-1315/121/5/052066.

Zdanyte, K., \& Neverauskas, B. (2014). Ensuring of sustainable development for contemporary organizations development. Economics and management, 19(1), 120-128. DOI: 10.5755/j01.em.19.1.5737. 